\title{
ESTUDO DOS ACIDENTES NA INFÂNCIA EM UM PRONTO SOCORRO PEDIÁTRICO
}

Fernanda Rocha Fodor Filócomo ${ }^{1}$

Maria de Jesus C. Sousa Harada ${ }^{2}$

Conceição Vieira Silva ${ }^{3}$

Mavilde da L.G. Pedreira ${ }^{4}$

Filócomo FRF, Harada MJCS, Silva CV, Pedreira MLG. Estudo dos acidentes na infância em um pronto socorro pediátrico. Rev Latino-am Enfermagem 2002 janeiro-fevereiro; 10(1):41-7.

O objetivo deste estudo foi identificar os acidentes na infância registrados em um pronto socorro infantil. Os dados foram coletados entre janeiro e março de 1999, através de um questionário contendo dados sobre a identificação da criança e do acidente. A população constou de 942 crianças e a amostra ficou reduzida para 890. Principais resultados: a faixa etária mais atingida foi de 7-11 anos, sendo 56,1\% do sexo masculino e $43,9 \%$ feminino, o tipo de acidente mais freqüente foi queda (46,9\%), os pais estavam presentes em $43,4 \%$ dos casos. Em 95,7\% das ocorrências a criança recebeu alta hospitalar após o atendimento.

DESCRITORES: prevenção de acidentes, acidentes domésticos, enfermagem pediátrica

\section{STUDY OF ACCIDENTS INVOLVING CHILDREN ASSISTED AT AN EMERGENCY WARD}

The aim of this study was identify cases of accidents victimizing children assisted at the emergence ward of a general hospital. Data were collected from January to March, 1999 through a protocol with variables related to the identification of the accidents as well as of the children. The sample in the study consisted of 890 children of whom $56.1 \%$ were males and $43.9 \%$ were females. The most prevalent accident was fall (46.9\%), the age with a major incidence was between 7 and 11 years old, the parents were present to $43.4 \%$ of the cases. The children were discharged from hospital after being given care in $95.7 \%$ of the cases.

KEY WORDS: accident prevention, accidents at home, pediatric nursing

\section{ESTUDIO DE LOS ACCIDENTES EN LA INFANCIA Y REGISTRADOS EN UNA UNIDAD DE URGENCIAS PEDIÁTRICAS}

El objetivo de éste estudio fue el de identificar los accidentes infantiles registrados en una unidad de Urgencias Pediátricas (UUP). Los datos fueron recolectados en el periodo de Enero a Marzo de 1999, utilizando un cuestionario que contenía datos sobre la identificación del niño y del accidente. La población estuvo conformada por 942 niños y la muestra fue reducida para 890. Principales resultados: la faja etárea más afectada fue la de 7 a 11 anos, siendo 56,1\% del sexo masculino y 43,9\% femenino; el tipo de accidente más frecuente fue la caída (46,9\%), los padres estaban presentes en $43,4 \%$ de los casos. En 95,7\% de las ocurrencias, el niño recibió alta hospitalaria después de ser tendido.

DESCRIPTORES: prevención de accidentes, accidentes domiciliares, enfermería pediátrica

\footnotetext{
${ }^{1}$ Enfermeiro, Aluno do curso de Especialização em Enfermagem Pediátrica e Neonatal pela Universidade Federal de São Paulo; ${ }^{2}$ Enfermeiro, Professor Assistente, e-mail: jjharada@uol.com.br; ${ }^{3}$ Enfermeiro, Professor Adjunto; ${ }^{4}$ Enfermeiro, Doutor em Enfermagem. Departamento de Enfermagem Disciplina de Enfermagem Pediátrica da Universidade Federal de São Paulo
} 


\section{INTRODUÇÃO}

$\boldsymbol{O}_{\text {s acidentes na infância representam cada vez mais uma }}$ importante causa de morbi-mortalidade no mundo atual, constituindo um grande problema de saúde pública, ao lado de doenças gastrointestinais, infecções respiratórias e desnutrição protéicocalórica ${ }^{(1)}$. Segundo relatório da Organização Mundial da Saúde (OMS) divulgado no Jornal do Conselho Regional de Medicina de São Paulo, em junho de 1999, as lesões traumáticas são um grave problema de saúde, pois representaram $16 \%$ das doenças em escala mundial no ano de $1998^{(2)}$. No Brasil, as causas externas (acidentes, violência, etc.) constituem $19,5 \%$ da mortalidade e no grupo etário de 5-19 anos a principal causa de óbito ${ }^{(3)}$.

A convenção sobre os direitos da criança e do adolescente normatiza que, deve-se assegurar a todos os setores da sociedade, em especial aos pais e as crianças, o conhecimento dos princípios básicos de saúde e entre outros 0 de ações de prevenção de acidentes, recebendo apoio para a aplicação destes conhecimentos ${ }^{(4)}$.

Pelo exposto fica evidente a necessidade de investirmos na prevenção de acidentes, e a primeira etapa deste processo seria modificar o conceito de que os acidentes estão relacionados com casualidade e imprevisibilidade.

Para tanto, é necessário adotarmos o novo conceito, onde o acidente seria considerado como "injúria não intencional" causada pela transmissão rápida de um tipo de energia dinâmica, térmica ou química de um corpo a outro ocasionando danos e até a morte ${ }^{(5)} \mathrm{e}$, desta forma, podem ser evitados e controlados.

Paralelamente a este conceito, tem-se usado para descrever a epidemiologia dos acidentes o modelo agentehospedeiro-ambiente. $\mathrm{O}$ agente seria a forma de energia que lesa os tecidos orgânicos; o hospedeiro seria a criança onde identificaríamos o risco de acidente de acordo com o seu estágio de desenvolvimento e 0 ambiente incluiria a situação física e psicossocial na qual ocorre 0 acidente. 0 conhecimento desta tríade fornece subsídios para direcionar ações de prevenção e identificar os grupos de maior risco ${ }^{(3)}$.

Iniciativa importante dentro deste contexto foi a Campanha Nacional de Prevenção de Acidentes, lançada em outubro de 1998 pela Sociedade Brasileira de Pediatria, com o objetivo de fornecer indicadores de prevenção de acidentes.

Desta forma entendemos que é de importância indiscutivel para os enfermeiros pediatras a reflexão e a conscientização sobre a necessidade de engajamento neste processo, através da elaboração de programas de prevenção, tendo como base a identificação dos fatores de risco na população alvo.

Portanto, temos como principais objetivos neste estudo: - identificar os acidentes na infância atendidos em um pronto socorro pediátrico;

- fornecer subsídios que contribuam para a elaboração de um programa de prevenção de acidentes à população usuária da instituição pesquisada.

\section{MATERIAL E MÉTODO}

Caracterização do local pesquisado

A pesquisa foi desenvolvida em um Pronto Socorro Infantil (P.S.I.), de um hospital geral, que atende crianças de 0 a 12 anos de idade. Possui 06 leitos de retaguarda, sendo 01 de isolamento. A equipe de enfermagem é composta por sete enfermeiros e quatorze auxiliares de enfermagem. A equipe de pediatras é distribuída durante as 24 horas, com dois plantonistas por turno.

O Hospital tem caráter privado, caracterizado como de grande porte. É considerado de alta complexidade por possuir serviços de diagnóstico e terapêutica, unidade de terapia intensiva adulto, pediátrica e neonatal. Está situado no bairro de Higienópolis, no Município de São Paulo.

Tipo de estudo

A pesquisa trata-se de um Survey descritivo, transversal, sobre os casos de acidentes na infância, identificados por ocasião de seu atendimento num pronto socorro infantil. Optou-se pelo estudo do tipo descritivo pois permite descrever com exatidão os fatos e fenômenos de determinada realidade ${ }^{(6)}$.

População e amostra

A população foi constituída de 942 crianças, na faixa etária de 0 a 12 anos de idade, vítimas de acidentes, atendidas no referido P.S.I. no período de estudo, procedentes de diversas localidades do município de São Paulo. A amostra foi reduzida a 890 crianças vítimas de acidentes, em virtude de falhas no preenchimento do instrumento de coletas de dados.

Instrumento de coleta de dados

Foi elaborado um instrumento de coleta de dados, que continha grupos de variáveis que pretendiam caracterizar 3 aspectos: 1. dados de identificação da criança; 2. dados específicos sobre 0 acidente, como: tipo, local e quem estava presente no momento da ocorrência; 3. evolução do caso: alta ou necessidade de internação em unidades específicas e óbito (ANEXO).

Este instrumento foi previamente testado em um período de 30 dias, realizando-se as alterações necessárias.

Os dados deste estudo foram coletados no período de $1^{\circ}$ de janeiro a 31 de março de 1999, através do Boletim de Atendimento de Emergência (B.A.E.), aberto no momento de entrada da criança no P.S.I., onde constam dados sobre a identificação da criança e 0 diagnóstico de entrada. Este procedimento é feito rotineiramente na referida instituição e, através destes boletins, eram identificados os casos de acidentes. 
A partir desta identificação, foi realizada uma entrevista com o acompanhante da criança, pela enfermeira de plantão no momento do atendimento.

Para tanto, foi realizado um treinamento com todas as enfermeiras da unidade, previamente a coleta dos dados, enfocandose as finalidades da investigação e a metodologia utilizada.

Vale ressaltar, que a proposta deste estudo foi encaminhada para a Comissão de Ética da Instituição, vinculada ao estudo, sendo aprovada sua realização por estar de acordo com as normas estabelecidas, sob parecer de $n^{\circ} 473 / 99$.

Análise dos dados

Os dados foram tabulados através de planilha eletrônica, Microsoft Excel. Ressaltamos que todos os protocolos foram revisados pelas pesquisadoras antes de serem informatizados, com o objetivo de se evitarem erros de preenchimento no banco de dados. Foi utilizado Teste do Quiquadrado para análise das variáveis independentes, sendo estas o sexo e a idade da população investigada.

\section{RESULTADOS E DISCUSSÃO}

No presente estudo obtivemos nos meses de janeiro a março um total de 9686 atendimentos, sendo que destes, 942 (9,72\%) tiveram como causa acidentes, representando quase $10 \%$ de todos os atendimentos, o que é considerado bastante representativo frente às diversas possibilidades de patologias que estão presentes na população infantil, uma vez que estas ocorrências provavelmente eram passíveis de prevenção.

Tabela 1 - Crianças vítimas de acidentes na infância atendidas em um P.S.I., segundo sexo e idade. São Paulo, 1999

\begin{tabular}{ccccc}
\hline & \multicolumn{2}{c}{ Masculino } & \multicolumn{2}{c}{ Feminino } \\
Idade & $N$ & $\%$ & $N$ & $\%$ \\
\hline \& que 1 & 10 & 2,0 & 23 & 5,9 \\
1 a 3 & 159 & 31,9 & 106 & 27,1 \\
4 a 6 & 124 & 24,8 & 85 & 21,8 \\
7 a 11 & 194 & 30,9 & 171 & 43,7 \\
12 a 14 & 12 & 2,4 & 6 & 1,5 \\
\hline Total & 499 & 100 & 391 & 100 \\
\hline
\end{tabular}

$x^{2}=9,49$
De acordo com a Tabela 1, da totalidade da amostra do estudo (890 crianças), 499 (56,1\%) eram do sexo masculino e 391 $(43,9 \%)$ feminino. Estes dados são similares aos encontrados em estudo retrospectivo realizado com 260 crianças do município de Ribeirão Preto $(S P)^{(7)}$, onde obteve-se que $55,4 \%$ da população estudada era do sexo masculino e $44,6 \%$ feminino. Outro trabalho prospectivo com 270 crianças ${ }^{(8)}$, demonstrou que $67 \%$ eram do sexo masculino e $33 \%$ feminino. Dados similares também foram identificados em outros estudos da área ${ }^{(9-11)}$.

Este predomínio do sexo masculino, no que concerne aos acidentes na infância, é explicado, provavelmente, pela diferença de atividades desenvolvidas em cada sexo, estando o menino mais exposto às atividades dinâmicas que envolvem maior risco, enquanto meninas possuem atividades mais brandas. Outro fato importante é que, sócio-culturalmente, o menino adquire liberdade mais precocemente em relação às meninas e começam a realizar atividades com menor supervisão direta dos adultos, tendo, então, um maior tempo de exposição a situações que antecedem acidentes.

Quanto à idade das crianças verifica-se na Tabela 1, que a faixa etária mais atingida foi de 7 a 11 anos $(41,0 \%)$ seguidos de 1 a 3 anos $(29,8 \%)$. Dados semelhantes foram encontrados em estudo retrospectivo com 218 crianças, onde a prevalência mais significativa foi na faixa etária de 7 a 12 anos ${ }^{(11)}$. Nesta faixa etária existe uma predominância de ocorrências de acidentes, provavelmente, pelo fato da criança ainda não possuir completo domínio de noções como distância, velocidade, espaço e tempo, somando-se a estes fatores temos a supervisão inadequada do adulto responsável, o que pode favorecer uma maior exposição ao risco de tais ocorrências.

A Tabela 1 ainda nos permitiu verificar que a prevalência do sexo masculino foi entre a faixa etária de 1 a 11 anos, tendo ocorrido uma inversão desta predominância em menores de 1 ano. Nesta tabela, a análise estatística pelo Teste do Quiquadrado $\left(x^{2}=9,49\right)$ mostrou que é mais significante a ocorrência de acidentes no sexo feminino que no sexo masculino, na faixa etária que corresponde a crianças menores de 1 ano, constatação esta que pode ter relação com o desenvolvimento mais lento, tendência em andar e falar mais tardiamente em meninos do que meninas na faixa etária lactente $\mathrm{e}$ pré escolar ${ }^{(12)}$. 
Tabela 2 - Crianças vítimas de acidentes na infância atendidas em um P.S.I., segundo idade e tipo de acidente. São Paulo, 1999

\begin{tabular}{|c|c|c|c|c|c|c|c|c|c|c|c|c|}
\hline \multirow[b]{3}{*}{ Tipo } & \multicolumn{10}{|c|}{ Idade } & \multicolumn{2}{|c|}{ Total } \\
\hline & \multicolumn{2}{|c|}{$<1$ ano } & \multicolumn{2}{|c|}{1 a 3 anos } & \multicolumn{2}{|c|}{4 a 6 anos } & \multicolumn{2}{|c|}{7 a 11 anos } & \multicolumn{2}{|c|}{12 a 14 anos } & \multirow[b]{2}{*}{$\mathrm{N}$} & \multirow[b]{2}{*}{$\%$} \\
\hline & $\mathrm{N}$ & $\%$ & $\mathrm{~N}$ & $\%$ & $N$ & $\%$ & $\mathrm{~N}$ & $\%$ & $\mathrm{~N}$ & $\%$ & & \\
\hline Queda & 23 & 69,7 & 150 & 56,6 & 116 & 55,5 & 120 & 32,9 & 8 & 44,4 & 417 & 46,9 \\
\hline Contusẫo & 3 & 9,1 & 44 & 16,6 & 38 & 18,2 & 160 & 43,8 & 8 & 44,4 & 253 & 28,4 \\
\hline Ferimento & 2 & 6,1 & 23 & 8,7 & 29 & 13,9 & 30 & 8,2 & 2 & 11,2 & 86 & 9,7 \\
\hline Entorse & $z$ & $z$ & 4 & 1,5 & 7 & 3,3 & 31 & 8,5 & $z$ & $z$ & 42 & 4,7 \\
\hline Coppo estranho & $z$ & $z$ & 11 & 4,2 & 9 & 4,3 & 5 & 1,4 & $z$ & $z$ & 25 & 2,8 \\
\hline Queimadura & 2 & 6,1 & 12 & 4,5 & 3 & 1,4 & 5 & 1,4 & $\bar{z}$ & $z$ & 22 & 2,5 \\
\hline Pronaçẫo dolorosa & 1 & 3,0 & 11 & 4,2 & 1 & 0,5 & $z$ & $z$ & $z$ & $z$ & 13 & 1,5 \\
\hline Mordedura de animais & $z$ & $z$ & 3 & 1,1 & 3 & 1,4 & 7 & 1,9 & $z$ & $z$ & 13 & 1,5 \\
\hline Intoxicaçã̃o & 1 & 3,0 & 7 & 2,6 & 1 & 0,5 & $z$ & $z$ & $z$ & $z$ & 9 & 1,0 \\
\hline Atropelamento & $z$ & $z$ & $z$ & $z$ & 2 & 1,0 & 5 & 1,4 & $z$ & $z$ & 7 & 0,8 \\
\hline Outros & 1 & 3,0 & $z$ & $z$ & $z$ & $z$ & 1 & 0,3 & $\bar{z}$ & $z$ & 2 & 0,2 \\
\hline Colisônes auto & $z$ & $z$ & $z$ & $z$ & $z$ & $z$ & 1 & 0,3 & $z$ & $z$ & 1 & 0,1 \\
\hline Total & 33 & 100 & 265 & 100 & 209 & 100 & 365 & 100 & 18 & 100 & 890 & 100 \\
\hline
\end{tabular}

$N=$ freqüência absoluta

$\%$ = freqüência relativa

Com relação aos tipos de acidentes a Tabela 2 mostra que a maioria foi decorrente de queda $(46,9 \%)$. Na análise primária dos dados verificou-se que $254(60,9 \%)$ foram decorrentes de queda da própria altura e $39,1 \%$ de outros lugares: cama $(13,5 \%)$, bicicleta $(12,3 \%)$, escada $(9,2 \%)$, cadeira $(6,1 \%)$, muro $(6,1 \%)$ e outros. Logo a seguir aparecem com maior freqüência as contusões $(28,4 \%)$ e ferimentos (9,7\%). Dados semelhantes foram encontrados em estudo realizado na cidade de Ribeirão Preto, onde $46,92 \%$ dos acidentes foram decorrentes de queda, seguidos pelas contusões $(15,38 \%)$ e cortes $(11,92 \%)^{(7)}$. Em trabalho retrospectivo realizado com uma amostra de 2713 crianças, mostrou que $41,2 \%$ dos casos de traumatismos crânio-encefálicos foram decorrentes de quedas em crianças de 1 a 2 anos de idade ${ }^{(13)}$. Dados similares foram relatados em estudo realizado com 139 crianças, onde $30 \%$ das fraturas faciais identificadas foram devidas a quedas ${ }^{(14)}$.

Verificou-se também que a queda foi o tipo mais comum de acidente encontrado na faixa etária de menores de 1 ano e entre 1 a 3 anos, que se justifica pela fase de maturação motora, cognitiva e psicossocial onde encontram-se estas crianças, aprendendo a conhecer os seus limites e adaptando-se ao meio. Ainda em relação a quedas, estas permanecem em níveis elevados também em crianças acima de 4 anos, evento este relacionado provavelmente as atividades de lazer e esportes, próprios desta faixa etária, destacando-se jogos, bicicleta, patins e outros.

Com relação aos ferimentos $(9,7 \%)$, terceiro tipo de acidente mais identificado, acreditamos que estes estejam relacionados com a falta de habilidade motora para manusear objetos e utensílios pérfuro-cortantes, bem como, pelo fato de crianças estarem mais expostas a fatores de risco como cantos arquitetônicos e de mobiliários que muitas vezes encontram-se sem proteção.

Em relação a queimaduras a faixa etária mais atingida foi a menor que um ano (6,1\%), pois nesta idade as crianças estão, de acordo com a fase de desenvolvimento psicomotor, engatinhando e começando a dar os primeiros passos, têm muita curiosidade em explorar o espaço a sua volta e acabam aproximando-se de locais como fogão e ferro de passar roupa estando portanto, expostas a este tipo de acidente. Dados semelhantes foram identificados em dois outros estudos pesquisados demonstrando uma incidência para este tipo de acidente respectivamente de $7,69 \%$ e $6,8 \%^{(8,11)}$.

Vale ressaltar que a incidência encontrada na população deste estudo em relação a colisão, por autos e atropelamentos, é baixa quando comparada a demais estudos, devido provavelmente às condições sócio-econômicas elevadas da população deste trabalho, propiciando condições de moradia com menores fatores de risco, dentre os quais podemos referir moradia afastada de rodovias, educação de trânsito, utilização de equipamentos de segurança em automóveis entre outros.

Tabela 3 - Crianças vítimas de acidentes na infância atendidas em um P.S.I., segundo quem estava presente no momento da ocorrência. São Paulo, 1999

\begin{tabular}{ccc}
\hline & \multicolumn{2}{c}{ Freqüência } \\
Quem estava presente & $N$ & $\%$ \\
\hline Pais & 396 & 43,4 \\
Amigos & 198 & 22,2 \\
Professora & 74 & 8,3 \\
Parentes & 23 & 2,6 \\
BabálEmpregada & 18 & 2,0 \\
Nấo registrado & 153 & 17,2 \\
Sozinho & 38 & 4,3 \\
\hline Total & 890 & 100 \\
\hline
\end{tabular}


Da amostra estudada, em $43,4 \%$ dos casos a mãe ou o pai estavam presentes no momento do acidente, em $198(22,2 \%)$ amigos, em $74(8,3 \%)$ a professora e em $38(4,3 \%)$ casos a criança estava sozinha, como demonstra na Tabela 3. Em 153 casos não foi possível obter tal informação. Cabe lembrar que a presença do adulto não impede que 0 acidente aconteça, talvez por desconhecimento de como evitá-lo ou ainda por não estar realizando uma supervisão direta, isto é, encontra-se presente durante as atividades de lazer das crianças, porém realizando outras atividades. Acreditamos então que, uma melhor orientação sobre a prevenção destes acidentes e uma supervisão mais rigorosa por parte dos responsáveis poderá contribuir para que este índice decresça.

Tabela 4 - Crianças vítimas de acidentes na infância atendidas em um P.S.I., segundo local da ocorrência do acidente. São Paulo, 1999

\begin{tabular}{ccc}
\hline & \multicolumn{2}{c}{ Frequêencia } \\
Local & $\mathrm{N}$ & $\%$ \\
\hline Casa & 399 & 44,8 \\
Escola & 150 & 17,7 \\
Via pública & 63 & 7,1 \\
Areas de Lazer & 61 & 6,9 \\
Outros & 16 & 1,8 \\
Nấo registrado & 193 & 21,7 \\
\hline Total & 890 & 100 \\
\hline
\end{tabular}

Quanto ao local do acidente, obtivemos 399 (44,8\%) ocorrências em casa, 158 (17,7\%) na escola, 63 (7,1\%) em via pública, 61 (6,9\%) em locais de lazer e 16 (1,8\%) em outros lugares. Em 193 casos não foi possivel obter tal informação. Dados semelhantes também foram encontrados em estudo que demonstrou que a maioria dos acidentes ocorreram em casa $(79,2 \%)$, seguidos de locais públicos $(11,7 \%)$ e apenas $5,8 \%$ aconteceram na escola ${ }^{(15)}$.

Verificamos que a maioria dos acidentes ocorreu no ambiente doméstico e isto deve-se ao fato deste ser considerado um local "perigoso" pois, encontramos objetos pérfuro-cortantes, fogão, janelas sem proteção, medicamentos e produtos de limpeza mal armazenados, animais domésticos, etc. Os pais devem ser alertados sobre estes perigos, adotando sistemas e mecanismos de segurança em casa, tais como: proteção nas janelas, cancelas nas extremidades das escadas, protetores de tomadas, cantoneiras, travas de segurança nos sanitários, detectores de fumaça, armazenamento de medicamentos e materiais de limpeza em locais altos e com fechadura, dentre outros, para minimizar tais ocorrências. Vale ressaltar que, apesar destes sistemas contribuírem com a prevenção de alguns acidentes, não dispensam a supervisão dos pais que devem estar cada vez mais atentos.
Tabela 5 - Crianças vítimas de acidentes na infância atendidas em um P.S.I., segundo a evolução do atendimento. São Paulo, 1999

Freqüềncia

\begin{tabular}{ccc} 
& \multicolumn{2}{c}{ Frequêencia } \\
Evoluçẫo do atendimento & $\mathrm{N}$ & $\%$ \\
\hline Alta & 852 & 95,7 \\
Internaçỗo em Enfermaria de Pediatria & 29 & 3,3 \\
Imternaçẫo em Semi-Intensiva & 4 & 0,5 \\
Intemaçẫo em UTI & 2 & 0,2 \\
Transferència & 2 & 0,2 \\
Evasẫo & 1 & 0,1 \\
\hline Total & 890 & 100 \\
\hline
\end{tabular}

De acordo com a Tabela 5, observamos que, da amostra identificada, apenas 35 casos $(4,0 \%)$ necessitaram de internação hospitalar, sendo 29 em enfermaria de Pediatria, 04 na unidade de Semi-Intensiva, 02 na unidade de Terapia Intensiva e 02 casos necessitaram de transferência. Este índice demonstra que o nível de gravidade, dos acidentes atendidos neste serviço, não é alto e que a maioria deles, após receber 0 atendimento, está em condições de alta, somente uma pequena parte precisa de cuidados mais específicos.

Segundo a American Heart Association (1997-1999), anualmente nos Estados Unidos da América os traumas pediátricos são responsáveis por cerca de 25.000 mortes, 600.000 admissões hospitalares e 16 milhões de atendimentos em departamentos de emergência, com custos diretos ultrapassando 7,5 bilhões de dólares $^{(16)}$.

\section{CONSIDERAÇÕES FINAIS}

A análise dos dados demonstrou que:

- a incidência de casos de acidentes atendidos no P.S.I. estudado corresponde a $10 \%$ dos 9686 atendimentos;

- o tipo de acidente mais freqüente foi queda (46,9\%);

- houve maior incidência de acidentes em crianças do sexo masculino $(56,1 \%)$;

- a faixa etária mais atingida foi de 7 a 11 anos de idade $(41,0 \%)$;

- a maioria dos acidentes ocorreu na residência $(44,8 \%)$ e na presença dos pais $(43,4 \%)$;

- 95,7\% das crianças atendidas receberam alta e apenas 4,0\% necessitaram de internação imediata.

Acreditamos que a redução da incidência de acidentes na infância pode ser alcançada mediante estudos regionalizados e com a implantação de programas de prevenção específicos.

Um dos pilares para a redução desta incidência esta pautada na educação, a qual requer a mobilização de vários 
segmentos da população, a fim de assegurar às crianças e famílias

o provimento de informações e tratamentos necessários que minimizem esta problemática.

O enfermeiro é um educador, estando apto para realizar programas educacionais que envolvam pais e crianças através da conscientização da necessidade de prevenção de acidentes. Para o exercício desta função, acreditamos que as enfermeiras que trabalham em ambulatórios, creches, escolas e centros de saúde encontram-se em posição mais propícia para a implementação de programas de prevenção. Todavia, é possível e necessário inserir também as enfermeiras que trabalham no ambiente hospitalar neste contexto, utilizando este período de permanência como estratégia de desenvolvimento destes programas, que devem dirigir-se inicialmente aos pais, com temas que englobam desde 0 conhecimento sobre o desenvolvimento neuro-psico-motor da criança, sua relação com os tipos de acidentes, as principais noções de segurança, até a necessidade de uma supervisão mais efetiva.

Estas considerações estão imbricadas com as várias mudanças de paradigma que se têm observado no atendimento hospitalar, onde se destaca a educação continuada em saúde, entendendo-se as altas hospitalares como processos de transição, visando a qualidade de vida da população atendida.

\section{ANEXO}

\section{INSTRUMENTO DE COLETA DE DADOS}

N. ${ }^{\circ}$

1- Data da ocorrência:

2- Data do atendimento:

4- Idade:

$1-<$ que 1 ano

2- $1|-| 3$ anos

3- $4|-| 6$ anos

4- $7|-| 11$ anos

5- $12|-| 14$ anos

5- Procedência: (bairro, cidade)

6- Mês do atendimento:

7-.Tipo de acidente:

1- ferimentos agente

2- queimaduras

agente:

3-intoxicações agente:

4- quedas
RA:

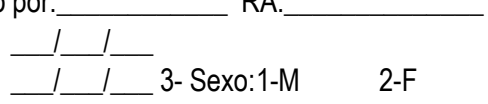

- atropelamentos

7- presença de corpo estranho onde

8- Quem estava presente no momento do acidente:

1- mãe

2- pai

3- babá

4- professora

5- outros

9- Local de ocorrência:

1- residência

2- escola

3- via pública

4- outros

10- Evolução do caso:

1- alta

2- internação:

1- UTI

2- Semi- intensiva

3-Enfermaria de Pediatria

3- óbito

4- transferência motivo

11- Observações

\section{REFERÊNCIAS BIBLIOGRÁFICAS}

1. Del Ciampo LA. Estudo dos acidentes na infância na área de Vila Lobato. [tese]. Ribeirão Preto (SP): Faculdade de Medicina de Ribeirão Preto/USP; 1994

2. Mestreneo R. OMS denuncia aumento de mortes por lesões traumáticas. Cremesp 1999 junho; (142).

3. Maciel W. Campanha nacional de prevenção de acidentes na infância e adolescência. Bol Soc Ped 1998 nov/dez; (73):4-5.

4. Convenção sobre os Direitos da Criança e Adolescente de 1990. Decreto n 99.710. Diário Oficial da União, 1990. 22 nov.

5. Blanc D. Conceitos básicos e aspectos preventivos gerais. In: Blanc $D$, organizador. Prevenção de acidentes na infância e adolescência. São Paulo (SP); 1994. p. 2-13.

6. Triviños ANS. Introdução à pesquisa em ciências sociais: a pesquisa qualitativa em educação. São Paulo: Atlas; 1987.

7. Del Ciampo LA, Ricco, RG, Muccillo, G. Acidentes: sabemos preveni-los? Pediatria 1997 julho/dez; 9(4): 263-6.

8. Matos MAG, Silva GAP, Ferreira CRP, Teixeira MLPD. Perfil epidemiológico das crianças internadas por acidentes no hospital da restauração. PIBIC [online] out 1996. Avaliable from: URL:http:/ propesq.ufpe.br/anais/ccs.03htm.

9. Carter $\mathrm{YH}$, Jones PW. Accidents among children under five years old: a general practice based study in north Staffordshire. Br J Gen Practice 1993 April; 43: 159-63.

10. Forlin E, Marchezini EJ, Ramos CH, Falavinha R. Aspectos epidemiológicos do trauma em crianças. Rev Bras Ortop 1995 out; 30 (10):761-4. 
11. Harada MJCS, Botta MLG, Kobata CM, Szauter IH, Dutra G, Dias EC. Epidemiologia em crianças hospitalizadas por acidentes. F Med (Br) 2000 out-dez; 119(4):43-7.

12. Gesell A. Cada criança é um indivíduo. In: Gesell A. A criança dos zero aos cinco anos. São Paulo: Martins Fontes; 1992. p. 29-43. 13. Freitas PEP, Oliveira QE, Nerung L, Henz DM, Camozzato A. Traumatismos cranioencefálicos acidentais em crianças: estudo de 2173 casos. AMRIGS 1990; 34(1): 19-23.
14. Anderson PJ. Fractures of the facial skeleton in children. Injury 1995 jan; 26 (1):47-50.

15. Alpert JJ, Guyer B. Clínicas Pediátricas da América do Norte. Rio de Janeiro: Interamericana; 1985.

16. Chamides L, Hazinski MF. Pediatric Advanced Life Suppport. Illinois: American Heart Association; 1999. 